\title{
HELIOLATITUDE REGULARITIES OF MAGNETICALLY DISTURBED DAYS WITH DAILY AVERAGE GEOMAGNETIC INDEX Dst $<-100$ nT
}

\author{
G.A. Makarov \\ Yu.G. Shafer Institute of Cosmophysical Research \\ and Aeronomy SB RAS, \\ Yakutsk, Russia,gmakarov@ikfia.ysn.ru
}

\begin{abstract}
This paper considers storm days for a period 1966-2015 when the daily average geomagnetic Dst index was $<-100 \mathrm{nT}$. The distribution of the number of days with a high daily average Dst is shown to depend on Earth's heliolatitude $\varphi$ : the number of days increases with the absolute value of $\varphi$ in both solar hemispheres. It is found, as expected, that the seasonal distribution of storm days with $D s t<-100 \mathrm{nT}$ has equinoctial maxima. Moreover, there is a noticeable increase in the number of such days in July and November. It is noted
\end{abstract}

that at Earth's heliolatitudes $4.1^{\circ}-5.0^{\circ}$ there is a sharp increase in the number of storm days. It is established that this increase occurs during storm events in July and November, which stand out against the seasonal distribution of highly disturbed days.

Keywords: geomagnetic Dst index, geomagnetic storm, seasonal variation of magnetic activity, Earth's heliolatitude.

\section{INTRODUCTION}

Geomagnetic storms result from the influence of disturbed structures in the solar wind on Earth's magnetosphere. They occur during an increase in solar activity when plasma streams from coronal holes and highspeed solar wind streams are ejected into interplanetary space. These formations in the solar wind usually have a southward $B_{\mathrm{z}}$ component of the interplanetary magnetic field (IMF). With this orientation of IMF, interplanetary and terrestrial magnetic fields reconnect, and the energy from the solar wind is transferred into Earth's magnetosphere [Akasofu, 1981]. In the magnetosphere, an electric field is generated which is directed across the tail from the dawn side to the dusk one, and the plasma is convected to Earth, thus increasing particle fluxes in radiation belts. The azimuthal circulation of ring current particles causes the horizontal component of Earth's magnetic field to decrease.

A quantitative measure of the ring current is the geomagnetic index Dst. This index also involves contribution from other current systems such as magnetopause currents and magnetic tail currents [Gonzalez et al., 1994; Feldstein et al., 2003]. It is determined from hourly average values of the horizontal geomagnetic field component, usually recorded at four low-latitude observatories. The recovery storm phase is characterized by the ring current decay [Yanovskii, 1978].

It has been found that strong geomagnetic storms are caused by the influence of interplanetary coronal mass ejections (ICMEs) and corotating interaction regions (CIRs) on Earth's magnetosphere. Detailed studies in this area have been carried out in [Ermolaev, Ermolaev, 2002; Yermolaev et al., 2017; Echer et al., 2008; Gonzalez et al., 1994, 2011; Gopalswamy, 2009; Watari, 2017]. ICMEs are divided into magnetic clouds and ejecta; among CIRs are sheaths. These structures have certain sets of interplanetary medium parameters. In a sheath at the front of fast and slow flows (CIR events) and ahead of the leading edge of ejecta (sheath events), the plasma has higher density and temperature, and the thermal pressure is higher than the magnetic pressure [Nikolaeva et al., 2011]. The magnetic cloud differs from ejecta, having a higher and more regular magnetic field. Very strong geomagnetic storms are generated by several interacting ICMEs [Yermolaev, Yermolaev, 2008].

There is a seasonal variation in geomagnetic storms with two peaks around the equinoxes. It is associated with axial and equinoctial mechanisms as well as with the Russell - McPherron mechanism [Echer et al., 2011]. At the same time, Mursula et al. [2011] note that substorm distribution and geomagnetic activity variation, characterized by the $A_{\mathrm{p}}$ index, have only one maximum per year in spring and autumn months depending on solar activity cycle. The annual variation of geomagnetic activity during intense geomagnetic storms with maximum in July is discussed in [Clúa de Gonzalez et al., 2002]. Clúa de Gonzalez et al. [2002] show that there is a peak in the annual distribution of strongly magnetically disturbed days in November.

In this paper, we consider regularities of the distribution of magnetically disturbed days, characterized by daily average $D s t<-100 \mathrm{nT}$, when Earth's heliolatitude $\varphi$ changes, i.e. the heliolatitude of the Earth projection on the solar disk.

\section{EXPERIMENTAL DATA AND PROCESSING RESULTS}

We analyze cases when daily average Dst is below $-100 \mathrm{nT}$. We consider the period from 1966 to 2015 and use data from the World Data Center for Geomagnetism, Kyoto, Japan [http://wdc.kugi.kyotou.ac.jp/dstdir/index.html]. We have selected 114 days that meet this criterion; in 66 days of them there were storms or a storm phase during one day, and in 48 days there were storms or storm families lasting for two days or more. Information on interplanetary medium and solar 
activity parameters has been taken from OMNI [http://omniweb.gsfc.nasa.gov] and SIDC databases [http://sidc.oma.be]. During this period there were no satellite measurements of plasma parameters for 14 days, of magnetic field parameters for 11 days; furthermore, there were no measurements of plasma and magnetic field parameters for 8 days.

It should be noted that after daily averaging the sample has days containing strong, extreme, and outstanding storms, which are called "intense geomagnetic storms" in this paper. The average Dst index is often determined for different phases of geomagnetic storms. Figure 1 exemplifies variations in hourly average Dst on some days. In the first case $(a)$ there is a sequence of two intense storms and three daily average daily Dst values are lower $-100 \mathrm{nT}$; in the second case $(b)$, the daily average Dst values are determined for the storm recovery phase lasting more than two days; in the third case $(c)$, all phases of the storm are analyzed.

The seasonal distribution of daily average $D s t<-100$ nT is shown in Figure 2. You can see that during a year it is non-uniform - a higher frequency of occurrence of minimum Dst is observed in spring and autumn (equinoctial maxima). In addition, a large number of events occur in July and November. Extreme values of

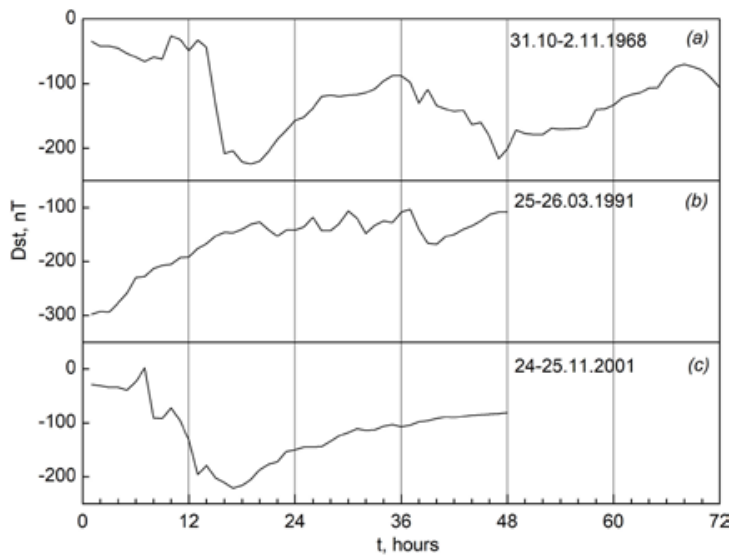

Figure 1. Variations in hourly average Dst: October $31-$ November 2, 1968 (a); March 25-26, 1991 (b); November 24 25, $2001(c)$. The time is measured from the first hour of UT for the first day of the given period

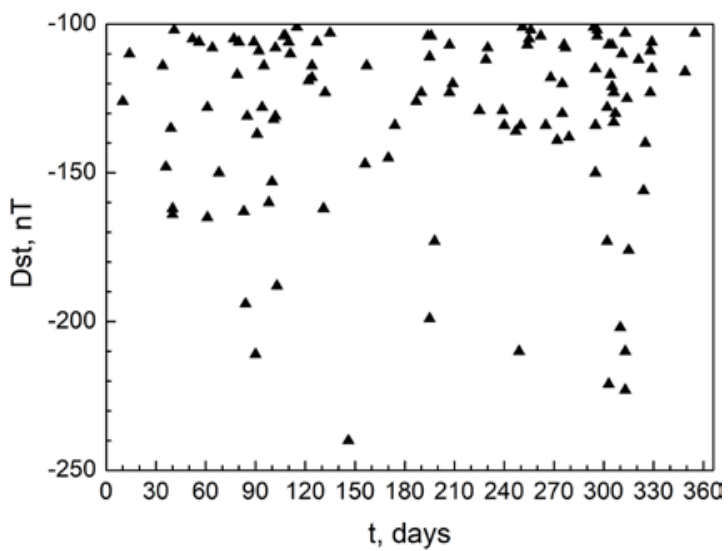

Figure 2. Seasonal distribution of daily average $D s t<-100 \mathrm{nT}$ Dst are recorded in spring: April 13, 1981 (-188 nT), March 25, 1991 (-194 nT), March 31, 2001 (-211
nT), May 26, 1967 (-240 nT); in autumn: September 06, 1982 (-210 nT), October 29, 1991 (-173 nT), October 30, 2003 (-221 nT, Hallowing event), November 06, 2001 (-202 nT), November 08, 2004 (210 nT), November 09, 1991 (-223 nT), November 10, 2004 (-176 nT); and in July: July 14, 1982 (-199 nT) and July 16, 2000 (-173 nT, Bastille Day event). Thus, the number of storm days with daily average Dst $<-100 \mathrm{nT}$ has a seasonal variation with maxima during the equinoxes. It should be noted that there is also an increase in the number of storm days in July and November.

Figure 3 shows the distribution of the number of days $n$ with daily average $D s t<-100 \mathrm{nT}$ as a function of Earth's heliolatitude $\varphi$. It can be seen that the number of days $n$ increases with the absolute value of $\varphi$ in both solar hemispheres. The number $n$ is maximum at extreme $\varphi$. It should be noted that there is a sharp increase in $n$ when Earth's heliolatitudes are $4.1^{\circ}-5.0^{\circ}$.

\section{DISCUSSION}

The obtained seasonal distribution of strongly disturbed days (see Figure 2) coincides with analogous intensity distribution of strong geomagnetic storms [Echer et al., 2011]. The spring increase in $n$ occur in March-April; the autumn one, in October-November, with a particularly large increase in November [Clúa de Gonzalez et al., 2002]. The increasing $n$ in July for intense storms is discussed in [Echer et al., 2011; Gonzalez et al., 2011].

In Figure 3, there is a pronounced peak at Earth's heliolatitudes $4.1^{\circ}-5.0^{\circ}$. This peak contains storm days from July 13 to 17 and from October 29 to November 3, i.e. the storm days standing out against the Dst seasonal variation in Figure 2.

The distributions of the number of days $n$ with daily average Dst $<-100 \mathrm{nT}$ as a function of Earth's heliolatitude $\varphi$ (Figure 3 ) and during a year (Figure 2) are likely to reflect the influence of two mechanisms of semiannual distribution of storm days: the axial mechanism, according to which with its maximum heliolatitudes Earth projects on the most active latitudes of the Sun [Cortie, 1912], and the equinoctial mechanism associated

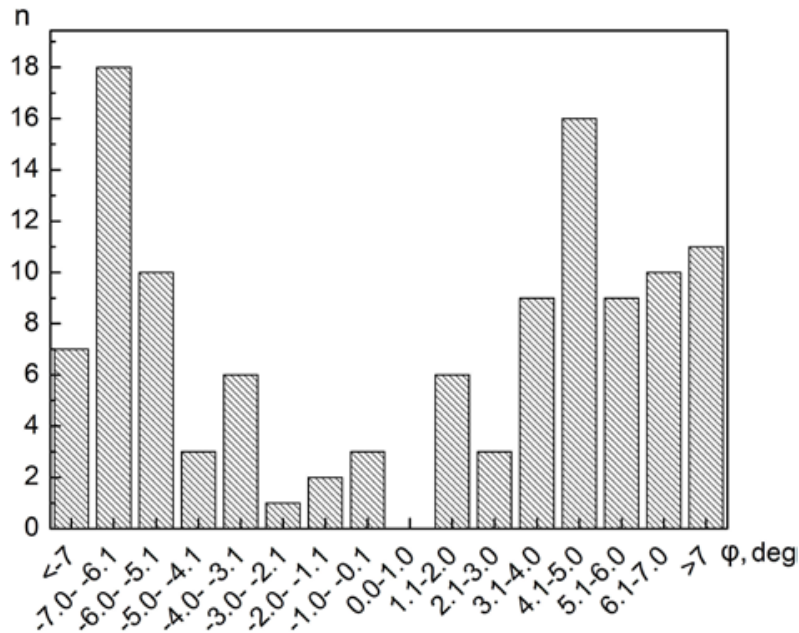

Figure 3. Distribution of the number of days $n$ with daily average $D s t<-100 \mathrm{nT}$ as a function of Earth's heliolatitude $\varphi$ 
with an increase in the interaction between the solar wind and the magnetosphere when the angle of attack is $90^{\circ}$ [Bartels, 1932]. Phases of the semi-annual variations determined by these two mechanisms are similar: $\varphi$ reaches extreme values on March 5-7 and September 6-8, the angle of attack takes on critical values on March 21 and September 22. This gives grounds to believe that both the mechanisms work simultaneously, complementing each other.

Figure 4 depicts variations in the solar wind velocity $V$, IMF modulus $|B|$, and values of IMF northwardsouthward component $B_{\mathrm{n}}$ as a function of Earth's heliolatitude, calculated for our sample. The values obtained for the interplanetary parameters differ significantly from the typical values [Kovalenko, 1983] upward. It can be seen that all the three parameters are distributed fairly evenly over the heliolatitude, $V$ features high values and averages about $620 \mathrm{~km} / \mathrm{s},|B|$ also has increased values and is $\sim 16 \mathrm{nT}, B_{\mathrm{n}}$ is generally southward and varies about the mean value of $-2.4 \mathrm{nT}$. The sharp increase in the values of all the parameters in the range of heliolatitudes $\left(-3.0^{\circ} \div-2.1^{\circ}\right)$ is a special case, because there was only one event.

Plots in Figure 4 suggest that the storms on the days considered were generated by disturbed solar wind streams. Their parameters virtually coincide with those obtained in [Uwamahoro, McKinnell, 2013] from the analysis of intense geomagnetic storms. Indeed, we have

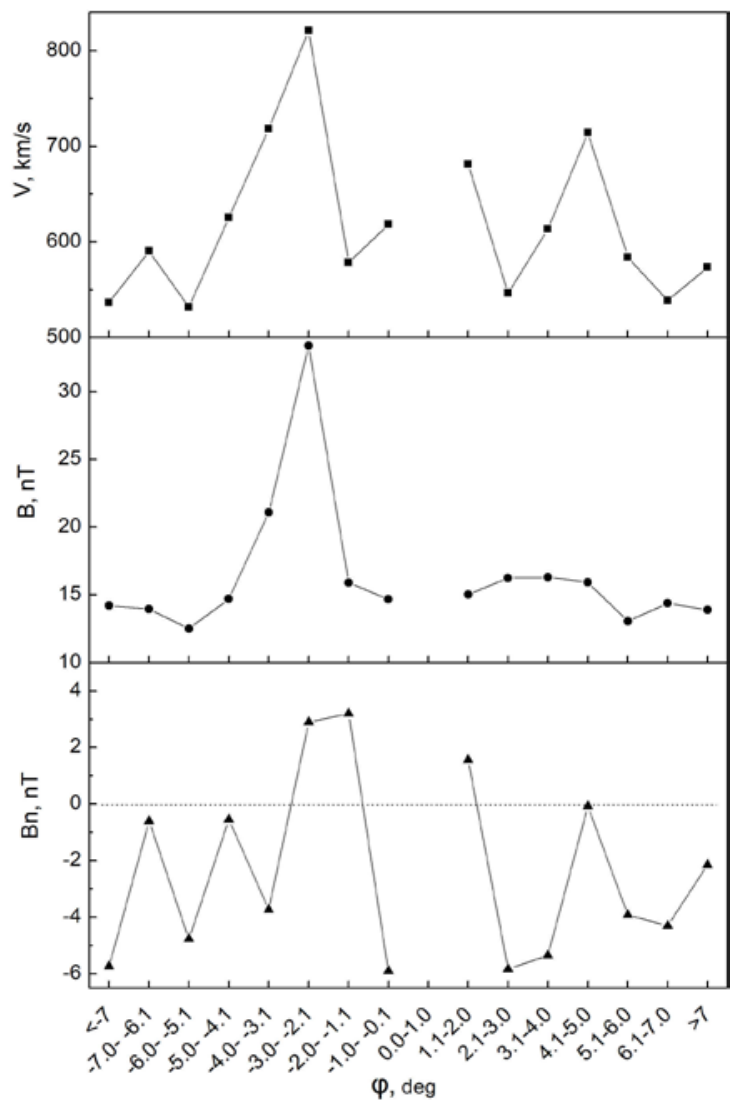

Figure 4. Variations in the solar wind velocity $V$, IMF modulus $|B|$, and values of IMF northward-southward component $B_{\mathrm{n}}$ as a function of Earth's heliolatitude $\varphi$

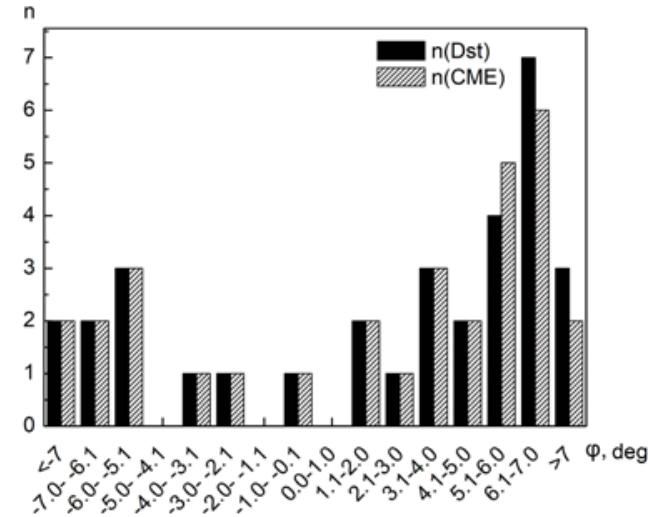

Figure 5. Distribution of the number of days with daily average Dst $<-100 \mathrm{nT}$ and the number of CMEs as a function of Earth's heliolatitude $\varphi$

selected days with daily average Dst $<-100 \mathrm{nT}$, which include major or recovery phase of intense geomagnetic storms.

To analyze the connection between these days with daily average $D s t<-100 \mathrm{nT}$ and plasma streams in the solar wind, we have used the catalog [Uwamahoro, McKinnell, 2013] that identifies interplanetary sources of geomagnetic storms in solar cycle 23 . There are 32 events from our sample in the catalog. Figure 5 shows the distribution of the number of days $n$ with daily average $D s t<-100 \mathrm{nT}$ and the number of CMEs as a function of Earth's heliolatitude $\varphi$. It is obvious that, as in Figure 3, the number of days with daily average $D s t<-$ $100 \mathrm{nT}$ increases with $\varphi$ in both solar hemispheres; moreover, in the northern hemisphere, the number of events far exceeds that in the southern one (22 and 10 respectively). Virtually the same regularity shows up in the distribution of the number of CMEs. Figure 5 shows the total number of CMEs without separation into types. The correlation coefficient between the distributions in Figure 5 is 0.95 . We can assume that in solar cycle 23 our events were caused by coronal mass ejections.

\section{CONCLUSION}

The following results have been obtained.

1. Distribution of the number of days $n$ with daily average $D s t<-100 \mathrm{nT}$ depends on Earth's heliolatitude $\varphi: n$ increases with the absolute value of $\varphi$ in both solar hemispheres and peaks at extreme values of $\varphi$.

2 . The distribution of the number of days $n$ with daily average $D s t<-100 \mathrm{nT}$ sharply increases at Earth's heliolatitudes $4.1^{\circ}-5.0^{\circ}$. This increase was caused by the storms occurring from July 13 to 17 and from October 29 to November 3, standing out against the seasonal distribution of strongly disturbed days.

3. As expected, the seasonal distribution of strongly disturbed days has equinoctial maxima; furthermore, it is worth noting that the number of such days increases in July and November.

4. From the catalog of solar and interplanetary sources of geomagnetic storms [Uwamahoro, McKinnell, 2013] a conclusion has been drawn that in solar cycle 23 the storms with daily average Dst <-100 nT were generated by coronal mass ejections. 


\section{REFERENCES}

Akasofu S.-I. Energy coupling between the solar wind and the magnetosphere. Space Sci. Rev. 1981, vol. 28, iss. 2, pp. 121-190. DOI: 10.1007/BF00218810.

Bartels J. Terrestrial magnetic activity and its relations to solar phenomena. Terrestrial Magnetism. 1932, vol. 37, pp. 1-52.

Clúa de Gonzalez A.L., Silbergleit V.M., Gonzalez W.D., Tsurutani B.T. Irregularities in the semiannual variation of the geomagnetic activity. Adv. Space Res. 2002, vol. 30, iss. 10, pp. 2215-2218.

Cortie A.L. Sunspots and terrestrial magnetic phenomena, 1898-1911. Monthly Notices of the Royal Astronomical Society.1912, vol. 73, pp. 52-60.

Echer E., Gonzalez W.D., Tsurutani B.T., Clúa de Gonzalez A.L. Interplanetary conditions causing intense geomagnetic storms (Dst $\leq-100 \mathrm{nT})$ during solar cycle 23 (1996-2006). J. Geophys. Res. 2008, vol. 113, A05221. DOI: 10.1029/2007 JA012744.

Echer E., Gonzalez W.D., Tsurutani B.T. Statistic studies of geomagnetic storms with peak $D s t \leq-50 \mathrm{nT}$ from 1957 to 2008. J. Atmos. Solar-Terr. Phys. 2011, vol. 73, iss. 11-12, pp. 1454-1459. DOI: 10.1016/j.jastp.2011.04.021.

Feldstein Y.I., Dremukhina L.A., Levitin A.E., Mall U., Alexeev I.I., Kalegaev V.V. Energetics of the magnetosphere during the magnetic storm. J. Atmos. Solar-Terr. Phys. 2003, vol. 65, iss. 4, pp. 429-446. DOI: 10.1016/S13646826(02)00339-5.

Gonzalez W.D., Joselyn J.A., Kamide Y., Kroehl H.W., Rostoker G., Tsurutani B.T., Vasyliunas V.M. What is a geomagnetic storm? J. Geophys. Res. 1994, vol. 99, pp. 57715792. DOI: 10.1029/93JA02867

Gonzalez W.D., Echer E., Tsurutani B.T., Clúa de Gonzalez A.L., Lago A.D. Interplanetary origin of intense, superintense and extreme geomagnetic storms. Space Sci. Rev. 2011, vol. 158, pp. 69-89. DOI: 10.1007/s11214-010-9715-2.

Gopalswamy N. Halo coronal mass ejections and geomagnetic storms. Earth, Planets and Space. 2009, vol. 61, pp. 1-3. DOI: 10.1186/BF03352930.

Kovalenko V.A. Solnechnyi veter [Solar Wind]. Moscow, Nauka Publ., 1983. 271 p. (In Russian).

Mursula K., Tanskanen E., Love J.J. Spring-fall asymmetry of substorm strength, geomagnetic activity and solar wind: implications for semiannual variation and solar hemispheric asymmetry. Geophys. Res. Lett. 2011, vol. 38, L06104. DOI: 10.1029/2011GL046751.
Nikolaeva N.S., Yermolaev Yu.I., Lodkina I.G. Dependence of geomagnetic activity during magnetic storms on the solar wind parameters for different types of streams. Geomagnetism and Aeronomy. 2011, vol. 51, no. 1, pp. 49-65. DOI: 10.1134/S0016793211010099.

Nikolaeva N.S., Yermolaev Yu.I., Lodkina I.G. Dependence of geomagnetic activity during magnetic storms on the solar wind parameters for different types of streams. Geomagnetism and Aeronomy. 2011, vol. 51, no. 1, pp. 49-65. DOI: 10.1134/S0016793211010099

Uwamahoro J., McKinnell L.-A. Solar and interplanetary precursors of geomagnetic storms in solar cycle 23. Adv. Space Res. 2013, vol. 51, iss. 3, pp. 395-410. DOI: 10.1016/j. asr.2012.09.034.

Yanovskii B.M. Zemnoi magnetizm [Terrestrial magnetism]. Leningrad, Leningrad State University Publ., 1978. 592 p. (In Russian).

Yermolaev Yu.I., Yermolaev M.Yu. Statistical relationships between solar, interplanetary, and geomagnetospheric disturbances, 1976-2000. Cosmic Res. 2002. vol. 40, no. 1, pp. 1-14.

Yermolaev Y.I., Yermolaev M.Y. Comment on "Interplanetary origin of intense geomagnetic storms (Dst $<-100 \mathrm{nT})$ during solar cycle 23'” by W. D. Gonzalez et al. Geophys. Res. Lett. 2008, vol. 35, L01101. DOI: 10.1029/2007GL030281.

Yermolaev Y.I., Lodkina I.G., Nikolaeva N.S., Yermolaev M.Y., Riazantseva M.O. Some problems of identifying types of large-scale solar wind and their role in the physics of the magnetosphere. Cosmic Res. 2017, vol. 55, no. 3, pp. 178-189. DOI: 10.1134/S0010952517030029.

Watari S. Geomagnetic storms of cycle 24 and their solar sources. Earth, Planets and Space. 2017, vol. 69, article 70. DOI: $10.1186 / \mathrm{s} 40623-017-0653-\mathrm{z}$.

URL: $\quad$ http://wdc.kugi.kyoto-u.ac.jp/dstdir/index.html (accessed April 12, 2018). 2018).

URL: http://omniweb.gsfc.nasa.gov (accessed April 12,

URL: http://sidc.oma.be (accessed April 12, 2018).

\section{How to cite this article}

Makarov G.A. Heliolatitude regularities of magnetically disturbed days with daily average geomagnetic index Dst<-100 nT. SolarTerrestrial Physics. 2018. vol. 4, iss. 3, pp. 20-23. DOI: 10.12737/stp343201803. 